\title{
Mindfulness-Based Cognitive Therapy: Primary Care Patients' Experiences of Outcomes in Everyday Life and Relapse Prevention
}

\author{
Josefine L. Lilja ${ }^{1 *}$, Malin Broberg1, Torsten Norlander ${ }^{2,3}$, Anders G. Broberg1 \\ ${ }^{1}$ Institute of Psychology, University of Gothenburg, Gothenburg, Sweden \\ ${ }^{2}$ Department of Clinical Neuroscience, Karolinska Institute, Stockholm, Sweden \\ ${ }^{3}$ Center for Research and Development, Evidens University College, Gothenburg, Sweden \\ Email: ${ }^{*}$ josefine.lilja@vgregion.se
}

Received 21 January 2015; accepted 23 March 2015; published 26 March 2015

Copyright (C) 2015 by authors and Scientific Research Publishing Inc.

This work is licensed under the Creative Commons Attribution International License (CC BY).

http://creativecommons.org/licenses/by/4.0/

(c) (1) Open Access

\begin{abstract}
The concept of mindfulness has attracted a growing body of research within behavioural medicine over the last decade. Mindfulness-based cognitive therapy (MBCT) has been developed to prevent relapse among people who suffer recurrent depression. Studies show impressive results, with a decrease in the risk of relapse of up to 50\%. However, primary care patients' own experiences and MBCT's effectiveness as a relapse program and aid to their ability to deal with everyday life remain relatively unexplored. The aim of the study, therefore, was to examine how primary care patients with recurrent depression perceive the usefulness of MBCT in preventing relapse. Nineteen patients who had participated in a MBCT program for recurrent depression within a primary care setting were interviewed 12 months after treatment. Qualitative thematic analysis was used to identify, analyse, and report patterns in the interviews. Analysis suggests two overarching themes, "Strategies for remission" and "Personal development". This study brings new information about what participants in MBCT classes describe as the most useful interventions for relapse prevention. The formal and informal meditation exercises focused on the body and the breath were described as the most important strategies for remission and the mindfulness practice gave the participants an enhanced self-knowledge that helped them to better deal with everyday stress and interpersonal functioning. The findings also indicate that traditional cognitive behavioural interventions, such as behaviour activation and establishing a maintenance plan, might not be as essential to relapse prevention as formerly thought.
\end{abstract}

${ }^{*}$ Corresponding author.

How to cite this paper: Lilja, J. L., Broberg, M., Norlander, T., \& Broberg, A. G. (2015). Mindfulness-Based Cognitive Therapy: Primary Care Patients' Experiences of Outcomes in Everyday Life and Relapse Prevention. Psychology, 6, 464-477.

http://dx.doi.org/10.4236/psych.2015.64044 


\title{
Keywords
}

\author{
Mindfulness-Based Cognitive Therapy, Primary Care, Qualitative Thematic Analysis, Relapse \\ Prevention
}

\section{Introduction}

Depression is one of the most common forms of mental health illness in the world and the most costly in Europe (Kaplan \& Laing, 2004). The prevalence rates of depression range from $3 \%$ to $10 \%$ across Europe (Ayseo-Mateos et al., 2001; Sobocki, Angst, Jönsson, \& Rehnberg, 2006). The total annual cost of depression in Europe was estimated at $€ 118$ billion in 2004 including the direct costs of outpatient care (€22 billion), drugs (€9 billion), and hospitalization ( $€ 10$ billion) and the indirect costs of morbidity and mortality estimated at $€ 76$ billion. Depression thus accounts for 33\% of the total cost of psychiatric disease in Europe (Sobocki et al., 2006). Those who recover from their first episode of depression have a 50\% risk of becoming depressed again, and among those with two or more depressive episodes, the risk for relapse is $70 \%$ to $80 \%$ (Angst, 1992). In as many as one in five patients depression becomes a chronic illness (Angst, 1988). Research has shown that mindfulness training can result in increased psychological and physical well-being (Baer, 2003, 2009; Brown \& Ryan, 2003; Nyklicek, 2010).

In 2002, Segal, Williams and Teasdale hoping to reduce the relapse rate by improving the content of cognitive behavioural therapy (CBT), created a CBT-maintenance program that mindfulness-based cognitive therapy (MBCT). They extracted two preventive components of CBT treatment: behavioural activation (which serves to break the depressive cycle of passivity) and decentring (which aims to change the function of dysfunctional thoughts). MBCT is based on the theoretical foundation that patients who have suffered several episodes of depression gradually become more vulnerable to relapse at times of low mood through the automatic activation of old habitual thought patterns. This has two important effects that reinforce each other. First, the patients engage in rumination about their mood, but find no effective way out of the depression. This then intensifies the negative affect, preventing effective problem-solving and leading to further depressive thoughts. Thus a self-destructive cycle is created, in which normal transient mood oscillation escalates into more serious depressive symptoms (Segal et al., 1996; Teasdale et al., 1995). Group participation in MBCT has been shown to reduce the risk of relapse by 50\% (Segal et al., 2002).

Most studies on the effect of mindfulness training have collected data through self-report questionnaires; as noted by Grossman (2008) "a number of intractable issues [...] continue to go unconsidered in investigations associated with the psychometric assessment of self-reported mindfulness” (p. 405). Qualitative studies of participants in mindfulness-based treatment programs therefore present an interesting research path towards obtaining new knowledge and deepening our understanding of the concept. Qualitative studies are particularly well-suited to studying unexplored phenomena and to improving our understanding of what is effective in treatment (Malterud, 2001). They can therefore be an important tool in developing a theoretical understanding of the psychological mechanisms at work in people who participate in MBCT and how these people use the class experience in their everyday lives to remain in remission.

Prior qualitative studies on participants' experiences of MBCT have focused mainly on therapeutic change and the development of mindfulness skills (Allen, Bromley, Kuyken, \& Sonnenberg, 2009; Bihari \& Mullan, 2012; Cebolla i Martí \& Barrachina, 2009; Mason \& Hargreaves, 2001). In 2011 Malpass and colleagues carried out a meta-ethnography of qualitative papers on MBCT and mindfulness-based stress reduction (MBSR) published since 2001 (Malpass et al., 2011). They conceptualized the way in which participants developed a new understanding of their illness over time and the role mindfulness had in helping them to manage their difficulties better. The final phase of change, "grounded flexibility" (Malpass et al., 2011: p. 70), is marked by transformation in the illness experience and the sense of self. Participants felt that they were better at taking action and more flexible in their responses; they had a greater sense of control and self-regulation, and more acceptance of themselves and their challenging thoughts and sensations; and finally, they had a sense of embodiment and command over the body that involved a new tolerance of physical difficulty and discomfort.

Bihari and Mullan (2012) suggested that MBCT participants have two parallel change processes of "relating 
mindfully” to themselves and to others. Allen and colleagues (2009) elucidated the psychological processes involved when participants' evaluate and describe MBCT as a treatment for recurrent depression. Their results showed four themes: control, acceptance, relationships, and struggle. They also showed that attending an MBCT class can enhance people's self-compassion and interpersonal relationships. The study highlights the participants' experiences and evaluations of the program and provides important clinical information that may help MBCT therapists to support and guide participants through obstacles in class.

In their long-term follow-up of MBCT participants, Munshi, Eisendrath and Delucchi (2012) found that the average number of minutes spent on mindfulness practice did not correlate with depression outcome, but that MBCT's effects might be more related to regularity of practice than to quantity. Langdon and colleagues (2011) interviewed 13 participants who had attended MBCT courses for an average of one year (range: 3.5 months to 4.5 years) before the study. They found that participants moved in and out of mindfulness practice over time, and that integration of mindfulness into lifestyle seems an important component of the continued practice cycle. They also found that cultivating mindfulness led to further intention to practice and to the development of more advanced mindfulness skills.

Michalak, Burg and Heidenreich (2012) stressed the importance of the body and the interaction between bodily, cognitive, and emotional processes in the recently emerging interdisciplinary research field of "embodiment”. They argue that all meditation exercises in MBCT are based on the development of a heightened awareness of the body, that the body has an important role in emotional expression and process in depression and is therefore a potent mechanism of action in MBCT.

None of the clinical studies cited above focused on MBCT as a long-term relapse-prevention program, nor on its utility in everyday life. The current study focused on the utility of MBCT in order to outline the relationships between mindfulness training, the most useful parts of MBCT for preventing relapse, and how participants transfer MBCT into everyday life.

\section{Aim and Research Questions}

The study aimed to examine how patients with recurrent depression perceived the usefulness of an MBCT program for preventing relapse of depression. The general research question, “After 12 months' participation in MBCT classes, how do patients describe their treatment?” was further refined to ask how MBCT

- functions to prevent relapse into depression?

- affects patients' ability to cope with everyday life?

\section{Method}

\subsection{Interventions}

The study was developed in a clinical setting within primary care that offers patients MBCT classes according to the MBCT program by Segal and co-workers (2002). Questions arose after the classes about what the participants had disclosed during class and in the follow-up about the MBCT program. Qualitative interviews were conducted 12 months after completion of MBCT treatment to examine the usefulness of MBCT in preventing relapse into depression.

The MBCT program consists of an 8-week group treatment in which 10 to 12 participants meet for 2.5 hours once a week (Lau \& Segal, 2007; Segal et al., 2002; Segal et al., 2013). After six sessions there is an additional silent meditation class. The sessions are a mix of psychoeducation and different types of meditation, yoga, and mindfulness exercises. The most common meditations used are body scanning, sitting (or "yoga”) meditation, and breathing space. Participants are also encouraged to practice daily mindfulness in their everyday life. This involves exercises in bringing full attention to everyday activities such as taking a shower or a walk, eating a meal, or talking with a friend. The classes also include traditional cognitive and behavioural interventions such as behavioural activation, recognition of negative automatic thinking and warning signs, and establishing a maintenance plan. The classes follow the same structure each week. First the participants do the exercise they have been practicing during the week before, then there is time to reflect, to engage in psychoeducation, and to learn CBT interventions. Finally a new exercise is introduced and homework is assigned (Lau \& Segal, 2007; Segal et al., 2002). 


\subsection{Data Collection and Participants}

The study was approved by The Regional Ethics Committee in Gothenburg (D.nr: 316-08). Participants were recruited at a primary health clinic in the western region of Sweden. Information about the study was given to the general practitioner at the clinic, who referred patients with recurrent depression to the study. Potential participants were screened and interviewed following criteria outlined in the Primary Care Evaluation of Mental Disorders (Prime-MD) (Spitzer et al., 1994) and the 4th edition of the Diagnostic Statistical Manual of Mental Disorders (DSM-IV) (American Psychiatric Association, 2002). The 38 patients who met the criteria for recurrent depression and were in full or partial remission were included in the MBCT class and signed an informed consent. The patients were instructed not change their medication during a 12 month period without notification to their general practitioners. There were 6 patients that dropped-out of class, 4 patients because their work schedule was not compatible with the class schedule and two because of private matters. The 22 who attended a minimum of 6 classes of the MBCT program were invited to participate in the interviews. In the 12-month re-assessment, all participants were screened and interviewed following Prime-MD and DSM-IV criteria. All participants were in full remission and no longer met the criteria for recurrent depression. One interview was excluded because the quality of the audiotape was poor, leaving 19 participants (17 women and 2 men) aged 30 to 68 years $(M=49)$ with complete qualitative data.

\subsection{Interview Procedure}

Interviews were conducted in a primary care setting in the Region of Västra Götaland, Sweden. Data were collected by three clinical psychologists, including the first author of this article. The interviews were semi-structured and the interview guide contained two major subject areas designed to elicit information about a) the patients' perceptions of how the treatment had affected the way they handled their everyday lives and b) their use of treatment components to prevent relapse into depression. The questions were open-ended to facilitate reflection, and probing questions were asked to elicit further exploration (Kvale \& Brinkmann, 2009). Interviews lasted for 30 to 50 minutes and were recorded using a digital voice recorder. The interviews were transcribed verbatim.

\subsection{Thematic Analysis and Procedure}

Thematic analysis (Braun \& Clarke, 2006) of the data set was used to identify, analyse, and report patterns in participants' descriptions of MBCT classes. Thematic analysis was chosen because it can be used with theoretical freedom and provides a valuable and flexible research tool. The method can provide general and detailed interpretation of data. Thematic analysis is not bound to a particular theory and can therefore be applied either inductively or deductively (Braun \& Clarke, 2006). In this study we analysed the data inductively ("bottom-up"), and thus coded the initial basic text with no a priori theoretical grid carried out the analysis on a semantic level (Braun \& Clarke, 2006). This decision was made with the aim of gaining information and research knowledge that matched the participants' own experience as closely as possible.

\subsection{Analysis and Interpretation}

The thematic analysis was conducted in the six phases suggested by Braun \& Clarke (2006). First, the researchers read all of the interviews to familiarize themselves with the depth and breadth of the content. Second, initial codes were generated by noting interesting features in the individual interviews. Third, all codes were gathered into potential themes (repeated patterns). In the fourth phase, we created a thematic map of the analysis to verify that the themes worked with the initial codes and across the entire data set. At this phase we had several potential themes such as "personal change", "coping strategies", "external impact”, "mindfulness as coping”, "mindfulness practice", "understanding of self/insight", and "setting boundaries". In the fifth phase we made a final refinement of the thematic map and the themes were divided into two different groups, Strategies for remission and Personal development. A few names were changed from the initial versions, for example the subtheme "understanding of self/insight" was changed to "personal self-knowledge and growth" to better demonstrate the content of the theme. In the sixth phase, producing the report, we chose data that captured the essence of the participants' experience, analysed the thematic map, and linked that data through our map to our research questions and points for discussion. 
The qualitative aspects of the research process were monitored by following Malterud's (2001) overall standards for qualitative inquiry concerning reflexivity, transferability, and shared assumptions of interpretation. In producing the report, rich quotations were selected and re-checked against the initial codes, final themes, research questions, and background literature to ensure that the final analysis was firmly anchored in every part of our material. The first and second authors (J. L. and M. B.) interpreted the data dialectically, moving between their pre-understandings and the data, and these interpretations were discussed until a consensus was reached on the formulation of the themes presented here. The software Nvivo10 was used to facilitate the analysis.

The authors' pre-understandings were based on several years of clinical and research work, experience, knowledge of clinical psychology, and the first author's experience as a practising CBT psychotherapist, all of which gave us a broad experience not only of human suffering, but also of people's ability to change and to learn how to cope with mental illness. Furthermore, the first author is a MBCT instructor and therefore has years of experience with yoga, meditation, and courses in mindfulness. The authors' pre-understandings can therefore be seen as both professional and well-grounded in the field. Additionally, the process of supervision and reflexive discussions with research colleagues helped the researchers to maintain reflexivity (i.e. awareness of preconceptions, clinical psychological theories, and personal investment).

\section{Results}

The analysis resulted in the identification of two overarching themes and subthemes (each composed of 2 or more categories; Figure 1). The theme Strategies for remission was divided into the subthemes "mindfulness practice" and "knowledge of sickness and health", and Personal development was divided into "intrapersonal development" and "interpersonal development”.
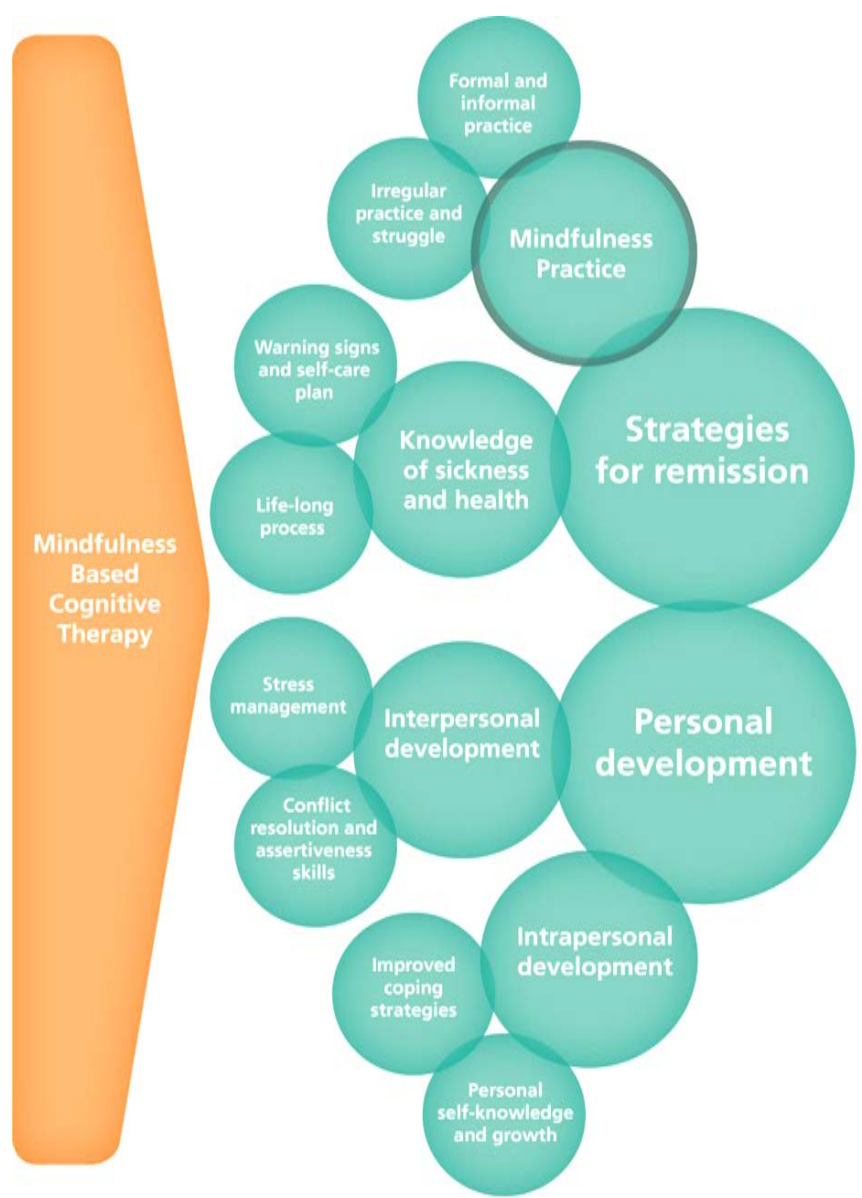

Figure 1. Two main overarching themes, Strategies for remission and Personal development, with subthemes and categories. 
The first theme, Strategies for remission, contains descriptions of the parts of MBCT that the participants still used and the strategies they had developed to remain in remission 12 months after the program ended. The second theme, Personal development, contains participants' accounts of what the therapy taught them, how they used mindfulness on a daily basis as a new way of dealing with life and mental distress, and how they developed and used new coping strategies. In quotations, participants are identified through an anonymized code to protect their anonymity while allowing readers to follow their individual stories.

\subsection{Strategies for Remission}

The analysis of the answers concerning strategies for remission resulted in two main subthemes, "mindfulness practice" and "knowledge of sickness and health". These subthemes revolved around important features of the program that the patients perceived as essential for their maintenance of lifelong mental health and learning to relate differently to their own health.

\subsubsection{Mindfulness Practice}

This subtheme revolved around the specific methods in MBCT that the participants found contributed to their initial changes and to their long-term well-being. Several participants said that the practice was the foundation of their ability to react adequately in stressful situations in everyday life. The mindfulness practice tended to be irregular and often included a mix of informal and formal exercises. The most formal meditation practices were breathing space, yoga meditation, and body scanning. The patients generally used a 3-minute session of breathing space to calm themselves in stressful situations and to step out of self-devaluating thinking. The yoga meditation and body scanning functioned to anchor themselves in the body and to make them more aware of early warning signs and their feelings in the moment.

\section{1) Formal and informal practice}

Most participants used both formal and informal practice on a weekly basis and had incorporated some sort of daily mindfulness exercise as an everyday habit. The formal practice was both a way to stay in touch with early warning signs from the body and a way to relax. Meditations that focused on breathing and the body seemed more frequently used and played an important role in helping participants to access information about their own emotional states. The practice also helped participants to better distinguish between things that were important and those that could be dismissed as unimportant.

I really use the short breaths ["breathing space"] a lot at my work-yes, I do. Then I do all the other exercises at home several times a week, but not always daily. But I notice in my body when I have rushed too much; the headache comes and I pull up my shoulders and feel in my body that I am tense. Then I think "Oh, now, I have not done my homework as I should have" and then I do it and then I do it daily, sometimes both in the morning and in the evening. It has really helped me to find the signals when I push myself too hard and need to calm down, and I use this to unwind and relax, to become more focused, and I notice that of the ten things I must do, I can weed out nine and just have one important thing left to do because I am doing these exercises. Ip 5

\section{2) Irregular practice and struggle}

Patients' practices were irregular and often more intense when their moods were lower and their stress higher. Some participants struggled with the practice and found it difficult to do their exercises, especially when their mood was in decline.

I can recognize when I feel bad, but then it's very difficult to get hold of this stuff; it's much easier when you feel good. It's hard, it feels more like I might wait it out until I feel a little better and then grab it. It is very difficult when I feel bad and am irritated and then I just don't give a shit about the whole thing somehow. Then I do not know if it is any use to me, but still it is there when I need it. Ip 4

It lowers your pace. I do the exercises for three or four days in a row and then I forget to do them because I feel good. And then it's okay some days, but every day I take a moment or two to do these breathing exercises when I work. Each week I do one of the exercises, but when I feel that my body is tense, I'll do the exercises for three or four days straight and then some days will pass before I do them again. Ip 5

\subsubsection{Knowledge of Sickness and Health}

Most participants reported that they now had knowledge and acceptance of their depression and a way to deal 
with it. They had learnt to see their management of depression as a life process and to know that there were ways to stay healthy and to prevent sickness. The participants had become aware of their early warning signs and had learnt how to act and take care of themselves in times of low mood.

\section{1) Lifelong process}

The participants said that they now realized that they had a lifelong mental health problem and felt that they were now equipped to deal with it. They had accepted that sensitivity to a lowering mood was a part of their lives that they had to handle on a daily basis.

I try to think about it as often as I can really, because you cannot just think about it when it is at its worst; it follows you the entire time. I'm trying to improve all the time, but it is small steps, nothing revolutionary. But I have to live with this for the rest of my life, to have with me these things and practice a little now and then. Ip 8

\section{2) Warning signs and self-care plan}

The participants' perceived themselves as more accepting of their low moods and felt they could handle their moods by slowing down, taking better care of themselves, and making small behavioural changes. None of the participants adhered regularly to the maintenance plan designed during the class to prevent relapse, but all stated that they had incorporated much of it in their daily thinking and behaviour. Most participants reported that they used exercises that involved the body as an early warning system. If they felt tension or pain in their body, they enhanced their mindfulness practice and self-nurturing activities. Some participants said that instead of using meditation or behavioural activation, they had a routine of daily activities that they maintained at a minimal level in times of lowering moods. This self-care plan served as an option to help them get through times of rumination and passive behaviour.

I certainly have the maintenance plan in mind somewhere, but I do not think about it. I have it there, inside myself somehow. I use the different tools from class. When I feel low, I cannot do much. I can only dedicate myself to my dog then. It's the only thing I have, I'm out walking, and taking care of the dishes or something, and I just keep walking. That's all. I can think it is no more than that. Those days are just "one of those days" and I leave it at that. Ip 3

\subsection{Personal Development}

The analysis of the answers relating to personal development and psychological change process resulted in two subthemes, "intrapersonal development" and "interpersonal development".

\subsubsection{Intrapersonal Development}

This subtheme deals with the personal psychological long-term changes that the participants described. They reported how, by using mindfulness in everyday life, they could take a step back when they were overwhelmed by emotional stimuli. They could observe and describe their emotions and negative cognitive processes and take a more balanced decision or action. The participants also noted that they had changed their ways of thinking, behaving, and handling emotions. Some participants described how the course had given them a different perspective on themselves and on how they lived their lives. The subtheme of intrapersonal development involved two categories, "improved coping strategies" and "personal self-knowledge and growth".

\section{1) Improved coping strategies}

The participants described how mindfulness training had helped them to take a step back and to stay calm in emotionally upsetting situations, to evaluate or redefine the situation, and to be more accepting and less judgemental in the aftermath of a situation. They found themselves more able to be aware in the moment and less reactive in their emotional expression. The participants reported how mindfulness training had made them more intentionally aware of the present moment. They described how they could calm down, stop their "mind-train", and catch themselves before their mood lowered or rumination took over.

It could be the acceptance- - that I reflect on and accept things or think about who it is that actually steers my life, what I want and whether it's for me or for others-things like that. That I relate to things in life in a different way. It could also be that I sit down and take a moment instead of just throwing myself down in a chair with a cup of coffee. Ip 7 
I reflected on that the other day. There have been situations in the past when I just reacted. But now, after it happened a few times, I could see it, so now I can take it in differently, relate to it in a different way. When that happens, I can see that my feelings are rushing ahead. I can see that it is not about me, but about the person, and then I can relate to it in a different way. When it begins to repeat itself, I see that I can sort each thing out. Ip 11

This category was further divided into three sub-categories "cognitive change”, "behavioural change”, and "emotional change" to better capture the content.

Cognitive change. The cognitive subcategory carried several descriptions with full and rich content. The participants gave detailed examples of how they had learnt to relate differently to cognitive stress by decentring and redirecting their thoughts. They had learnt how to deal with their habitual interpretation of situations and rumination. Instead of rumination, they learnt to focus first on taking a moment to assess the situation and then, if possible, to engage in problem-solving. Participants said that they reacted less to others' emotional expressions and felt less distressed when they took a mental time-out, thereby preventing the escalation of upsetting thoughts and rumination.

I'm aware of my thoughts and I can question them. I do not jump on this thought train and begin to ponder, but try to stay in the present. /.../ I do not magnify the thoughts. I do not put one thought after the other thought, but I try to remember that this is what is happening now, and that's it. Instead of what I used to do earlier, when I thought "then maybe that will happen and this will be so and so". Ip 4

Behavioural change. The behavioural subcategory comprised participants' experiences of making more active choices in life, of prioritizing behaviours that gave them energy (for example, yoga, mindful walking) and were nurturing and self-caring (for example, having a warm bath, taking a coffee break). They were also better at pacing their activity to match their level of energy.

Well my boss at work [noticed a change]. Only positive. She thinks that I have taken a step forward and dare to do things /... She thinks I have become stronger in myself somehow. I'm more positive and stand up for what I say, and do so more frequently. So I actually got a raise. Ip 2

If I have a bad day, I just think that this is not forever, it may just be today that it feels like this, and soon I'm good. It's usually only those thoughts I think, that I should sit still today or take it easy, and then hopefully it is good tomorrow. Instead of just speeding along, sometimes I just have to do that, too, but I'm much more aware of it. No, now it's like this today and then I put less “to-do's” on myself. Ip 6

Emotional change. The emotional subcategory had two main expressions. The participants were both more accepting of their emotions and better able to stay with the emotions without being overwhelmed. The exercises in MBCT taught them to reflect upon their feelings and gave them tools to verbalize them. They described themselves as more able to understand their emotions and use them as a way to get to know themselves and as a help and guide to behaviour when they needed to take care of themselves.

Well, I do not think I lose myself in the same way, but I've got the insight now that I can control some things on my own, and the biggest part is that I have learnt to stand alongside my troubles. I have a picture of myself standing next to a waterfall and looking at it, but not letting the emotions take over. Ip 10

\section{2) Personal self-knowledge and growth}

The pattern that emerged in the participants' stories was that they had often downplayed their own needs and placed too many demands on themselves. After MBCT, participants had a more secure and stable feeling about themselves, had learnt to set their own needs before others', and had lowered their demands on themselves. Some of the patients talked about how the program had led them to change their views on life; they were more open to seizing opportunities and had changed their perspectives on how to live their lives.

I've learnt to see that when I go over my boundaries I can easily get out of control and drain myself of energy. I've learnt that I have low impulse control, that I place too high demands on myself, and that I put the needs of others before my own. When I get into such a situation, I stop, I take a few deep breaths, and I think about what will happen to me if I let myself get caught up in the situation and just do what I think others expect. Ip 5 


\subsubsection{Interpersonal Development}

This subtheme centred on behavioural changes related to other people at work, at home, and elsewhere. Participants reported that they now handled stress and conflict more constructively. Several reported that the changes were noticeable enough to have been commented on by others and that MBCT had helped them to be more assertive and not so prone to avoid conflicts.

\section{1) Stress management}

Several participants reported having learnt about their automatic reactions in stressful situations and said that they now had an option for dealing with such situations. Mindfulness training helped them to stay in the moment, estimate the situation in a balanced way, act appropriately, and leave it at that. This behavioural conduct not only helped them during times of stress, but minimized their worry and rumination before and after stressful situations.

It can be used in many situations, and I think of situations at work, I use it a lot there. /.../ There are difficult situations at work, difficult patients to go to, so somehow, I think "now I am here and it's like this, and now I'm making the best of it". And I leave it when I go home instead of magnifying the situation and thinking about it on my way home like I used to before. Ip 4

Following MBCT, patients found they were able to calm themselves in stressful situations, evaluate the cause of the situation, and avoid letting their emotions take over and becoming reactive.

For example, at my work today, I'm working as a personal assistant, and when things happen and a person is not feeling well, I can stop and feel that it is not about me. I do my job. It's all about this person who is not feeling well, and I can stop and take a few deep breaths and feel that this does not affect me. I do not let emotions take over, I guess that's why. Ip 5

\section{2) Conflict resolution and assertiveness skills}

Several participants described how mindfulness had helped them to be more assertive and to face conflicts rather than try to avoid them. This effect was most noticeable in close relationships and in work relationships. The participants described how they could be clear about their own standpoint and stay present in a conflict situation instead of running away or escalating the conflict. They were also less judgemental of others' opinions and ideas.

The other day I knew a conflict with my ex was coming up. I was in the car when I got the call. I felt a peace in my body, knowing I could express myself. I thought about this using the traffic signals in CBT: red/yellow/green, though I say stop, think, talk. We were both tired and annoyed, but I could say that we should not be arguing about this now because we are both tired and stressed out and we do not want to argue. I could say it calmly and say that we are not doing this right now, so we can talk another day. It was a good feeling to have that control. I felt so happy and proud afterwards. Ip 11

Yes, my partner can still feel that I have a bad temper and all that, but I will stop in time before it feels like I have to make everyone agree with me, I think. Now I can let other people have their opinion and leave it at "so, you think the way you do". I can leave it when I have talked about how I feel about it all. If we do not really agree, I can leave without losing my mind, as I used to before. Ip 10

\section{Discussion}

The analysis of participants' descriptions of the utility of MBCT as a relapse prevention program resulted in the two main themes, Strategies for remission and Personal development. The formal and informal meditation exercises that focused on the body and the breath were the most important strategies for remission, and the mindfulness practice gave the participants increased self-knowledge that improved their coping strategies and interpersonal functioning. Our findings show that traditional behavioural and cognitive interventions such as behavioural activation and establishing a maintenance plan were missing in the interviews as potent ingredients in the participants' work to prevent relapse. All participants thought that MBCT had helped them to handle their mental health over time and had affected the way they handled their everyday stress in life.

This study goes beyond previous findings by illuminating not only the personal changes the patients perceived, but also which parts of the program the participants found important for these changes and useful in their everyday lives. This study therefore provides new knowledge on what patients perceive to be effective ingredients in 
MBCT for preventing relapse.

\subsection{Strategies for Remission}

The first research question, "How does MBCT function to prevent relapse?" can be seen as a response to Bihari and Mullan (2012). They found that MBCT participants expressed appreciation of the range of mindfulness practice, but that future research was needed to explore the specific practices in MBCT highlighted by the participants. The results of the present study contribute to our understanding of what makes MBCT a workable relapse therapy. The findings show that continued mindfulness practice, both formal and informal, is the most effective intervention against relapse. Overall, meditations involving the body (i.e., body scanning, yoga meditation, and breathing space) seem to be more accessible than most of the other MBCT exercises and are therefore more regularly used to train and cultivate mindfulness. Daily mindfulness exercises and keeping in contact with emotional signals from the body also seem to serve as important relapse interventions. A majority of the participants stated clearly that their mindfulness practice was both an anchor and a cornerstone of their continued mental well-being. These results are in line with those of Ingvarsson, Nordén \& Norlander, whose 2014 case study of MBCT participants indicated that the practice of mindfulness was the most important path towards healthy behaviours.

Mindfulness practice seemed to be a bridge over negative thoughts to personal development, improved coping strategies, and the progress of several cognitive-emotional and behavioural changes. That mindfulness practice, and more specifically practices that focused on the body and breathing, helped participants to tune into their emotional status supports the assumptions of Michalak and colleagues (2012) that the body has an important role in the emotional processes of depression and works as a potent mechanism of action in MBCT. Ingvarsson and colleagues (2014) also address the fact that MBCT participants become more aware of the body and its signals and come to understanding how the body can serve as a guide to distinguish between healthy and unhealthy behaviours.

Additional analysis of the participants' strategies for remission showed that their knowledge of sickness and health (i.e., their acceptance that recurrent depression is a lifelong mental health problem and their willingness to structure life around its management) was an important strategy to prevent relapse. The participants re-evaluated their knowledge of their own mental health and reappraised their cognitive and emotional experiences. Researchers have different opinions about whether cognitive restructuring takes place in mindfulness. Kabat-Zinn (2003), who founded the Mindfulness-Based Stress Reduction (MBSR) program, argued that no active reappraisal takes place and that the issue in mindfulness practice is only to mindfully and non-judgementally observe all experiences as they are and to then let them go. Nyklicek (2010) disputes this theory and argues that mindfulness practice involves reappraisal of prior knowledge concerning thoughts, emotions, and behaviours. The current study seems to support Nyklicek's view. Another hypothesis that needs further investigation is that although they share many components, the purposes of the MBSR and MBCT programs differ, in that MBCT targets dysfunctional thinking more specifically than MBSR does, and that one of the main aims of the MBCT program is to teach the participants to relate differently to their thoughts and emotions.

The present study also gives further response to Bihari and Mullan's (2012) question which practices to highlight or elaborate as strategies for remission. None of the participants actively used their maintenance plan and only a minority used traditional behavioural activation to handle their low moods. The maintenance plan was mostly "in the back of my head" or was described as "knowledge about my warning signs". It seems that these interventions from the MBCT were not prevalent 12 months after the course. None of the participants spoke spontaneously about psychoeducation or cognitive/behavioural interventions, only when they were specifically asked about them. These findings are somewhat puzzling because these interventions constitute the cornerstones of MBCT as a relapse prevention program (Segal et al., 2002). Our findings therefore suggest that there are other ingredients in the program, especially meditation practice, that are more potent strategies for remission and stable well-being than those that were intended to form the basis of the therapy.

\subsection{Personal Development}

Concerning the second research question, "How did the MBCT class affect participants' ability to cope with everyday life?” the analysis shows that the MBCT classes improved participants' strategies to handle everyday life and relationships (in the theme Personal development). The participants clearly stated that mindfulness 
training had improved their stress management and ability to handle conflicts. MBCT also gave the participants better personal self-understanding and they felt that they had grown as individuals (in the subtheme personal self-knowledge and growth). This result is in line with Carlson's 2013 hypothesis that mindfulness is a path to enhanced self-knowledge. Carlson (2013) reviewed evidence suggesting that mindfulness training improves people's understanding of themselves because it addresses two major barriers to self-awareness: a) informational barriers (the quantity and quality of information people have about themselves) and b) motivational barriers (i.e. protective motive that affect how people process information about themselves). Carlson further argues that prior efforts to improve individuals understanding of themselves have fallen short, either in studies of active behavioural feedback or introspection. Mindfulness training is different from self-focused interventions that involves, analysing, interpreting and explaining what is observed, and can therefore be seen as an enhanced form of self-knowledge because it teaches a person to both pay more attention to one's current experience and at the same time practice non-evaluative observation. This assumption is supported in the work by Lilja, Lundh, Josefsson, \& Falkenström (2012) who showed that experienced meditators were over-represented in clusters with higher levels of ability to observe and non-judge/non-react to inner and external experiences in a mindful way, when measuring levels of mindfulness. A majority of the non-meditating individuals, on the other hand, showed low levels of observing and high levels of non-judge/non-react to inner and external experiences (Lilja et al., 2012). Overall, we argue that these findings support the idea that enhanced self-knowledge, developed by mindfulness training, is an important ingredient in MBCT for relapse prevention.

\section{Discussion of Result in Conjunction with Previous Research}

Mindfulness has been described as increasing the ability to step out of negative self-judgemental thinking, and increase acceptance of low mood and feelings (Segal et al., 2002). Our findings, concerning Personal development, converge with this account. In the participants descriptions we could see a process that started with mindfulness practice. The practice gave the participants the powerful skill of being able to mindfully monitor their mental state and furthermore, handle everyday stress and demands in a non-judgemental way. Furthermore, this mindfulness training, gave them mental space to respond to difficult situations in a more a skilful cognitive, behavioural or emotional manner and, in turn, appears to mediate reductions in psychological symptoms and improvements in well-being. Result of the work is well in line with conclusions made by Baer (2009) "that the practice of mindfulness cultivates an adaptive form of self-focused attention that facilitates decentring and defusion; reduces rumination, fear of emotion, and avoidance of emotional stimuli; and improves the ability to engage in constructive, valued behaviour even while experiencing unpleasant thoughts and feelings” (p. 19).

Participants also reported that they could decentre and "turn towards" distressing thoughts, emotions and bodily sensations. This indicates that they had learned a more accepting way to deal with experiences. Accepting also in the sense of seeing themselves and their mental health as it really was, and thereby being able to make adequate behavioural adjustments accordingly. A greater acceptance of (emotional, cognitive and bodily) experience is thought to be a core change process of MBCT (Segal et al. 2002). As argued by Linehan (1993) and Hayes (2004) acceptance is not equal to stagnation or "giving up”, instead acceptance creates change.

Several of the subthemes are similar to those identified in previous qualitative studies; especially the theme of Intrapersonal development, which was noted in three previous studies (Mason \& Hargreaves, 2001; Finucane \& Mercer, 2006; Allen et al., 2009). The core construct “relating mindfully” found by Bihari \& Mullan (2012) also bear resemblance to our subthemes Interpersonal development, since it circle around how participants experience better interpersonal functioning and Intrapersonal development, that show patterns of improved individual coping strategies. The findings also support the hypothesis made by Malpass and co-workers (2011) that the participants final change phase is marked by transformation in the illness experience and the sense of self. When the themes were matched with the theoretical model that underpins MBCT, we found considerable correspondence, but also some parts in need of further elaboration, more specifically the behavioural and cognitive interventions. This raises some questions about how effective these interventions are as components of a relapse program, should those interventions be given less time during the classes, and if so, should the meditations exercises be enlarged?

Another way to look at the results is by focusing on the interaction and relationship between strategies for remission and personal development. The current study show that mindfulness practice is the foundation from which the change process develops in an evolving chain reaction and that it serves as an important strategy for 
remission. We propose that the mindfulness practice starts a personal development process that the participants experience is affecting their knowledge of themselves and thereby improving personal coping strategies and the way they deal with interpersonal conflicts. The practice further promotes acceptance of living with a lifelong mental health problem and the adjustments and techniques that are needed to stay well. The result of this process could be an evolving spiral that leads to a "mature mindful awareness". This is in line with the hypothesis proposed by Brown and Ryan (2003) who suggested that mindfulness practice leads to an evolving interconnection between self-regulation of behaviour and hedonic experiences, which enable meditators to act in greater accordance with their values and interest.

In sum, this study brings new information about what interventions participants in MBCT classes describe as most useful, 12 months after class ended. The formal and informal meditations exercises that focused on the body and the breath were describes as the most important strategies for remission and it seemed as if the mindfulness practice gave the participants an enhanced self-knowledge that helped them to better deal with everyday stress and interpersonal functioning. Additionally, the analysis show that the majority of the cognitive and behavioural interventions taught during MBCT class is not incorporated in the daily lives of the participants in follow-up interviews.

\section{Limitations and Future Research}

This study has some limitations. First, patients may have followed cultural script and gave the answers that they expected that we as psychologists wants, for example concerning how much they practiced meditation or in-depth answers of how the MBCT-class have affected their life. This might have been the case, but we argue that the diversity of the answers and that the interviewer probed for honesty, counterbalanced this issue. Second, patients may not on a conscious level (verbal) be aware of the change processes they have gone through. This might have influenced the content and the level of information that were provided. At the same time we wanted the information from the participants to be as close to their reality as possible and therefore kept the interview technique simple and on a semantic level. This provided us with the bottom-up perspective that we aimed for. The findings of this study should also be interpreted in the context of the sample, which represents people identified in primary care as suffering from recurrent depression. Since depression is one of the most common diagnoses in Swedish primary care we find it an interesting and valuable sample to study.

The results illuminate that further research is needed to explore in a controlled study to what amount and in what way participants use parts of MBCT, both the mindfulness-based and the cognitive and behavioural interventions, in order to secure and validate which impact the different interventions have. This could be accomplished by designing one group format that only practised the mindfulness exercises and left out the cognitiveand behavioural interventions, one group format were the participants only practiced cognitive and behavioural interventions and one control group in form of a traditional MBCT. The study illuminates the importance of the body in emotional processes in depression and as a potent relapse mechanism of action in MBCT. Further research should try to further investigate the role of the body in MBCT and if some adjustment and improvement in the MBCT program ought to be made, in order to improve its impact as a relapse program.

\section{Acknowledgements}

We would like to thank the participants for giving up their time to take part in interviews and for sharing their reflections. First author is ever so thankful to her colleagues Heléne Möller and Clara Zelleroth for their help with the interviews and MBCT classes. This study was financed by a research grant from the Vastra Gotalands County Council Research and Development Centre (FoUcentrum i Vastra Gotaland) and by support from the Tjorns Health Clinic and Head of Clinic Med dr. Torbjörn Erneholm.

\section{References}

Ayseo-Mateos, J. L., Vásques-Barquero, J. L., Dowrick, C., Lehtinen, V., Dalgard, O. S., Casey, P., Wilkinson, C., Lasa, L., Page, H., Dunn, G., Wilkinson, G., \& the ODIN Group (2001). Depressive Disorders in Europe: Prevalence Figures From the ODIN Study. British Journal of Psychiatry, 179, 308-316. http://dx.doi.org/10.1192/bjp.179.4.308

Allen, M., Bromley, A., Kuyken, W., \& Sonnenberg, S. J. (2009). Participants’ Experiences of Mindfulness-Based Cognitive Therapy: "It Changed Me in Just About Every Way Possible”. Behavioural and Cognitive Psychotherapy, 37, 413-430. http://dx.doi.org/10.1017/S135246580999004X 
American Psychiatric Association (2002). Mini-D IV. Diagnostiska kriterier enligt DSM-IV-TR. Danderyd: Pilgrim Press.

Angst, J. (1988). Clinical Course of Affective Disorders. In T. Helgason, \& R. J. Daly (Eds.), Depressive Illness: Prediction of Course and Outcome (pp. 1-48). Berlin: Springer. http://dx.doi.org/10.1007/978-3-642-73546-2_1

Angst, J. (1992). How Recurrent and Predictable Is Depressive Illness? In S. Montgomery, \& F. Rouillon (Eds.), Long-term Treatment of Depression (pp. 1-13). New York: John Wiley \& Sons.

Bihari, J. L. N., \& Mullan, E. G. (2012). Relating Mindfully: A Qualitative Exploration of Changes in Relationships through Mindfulness-Based Cognitive Therapy. Mindfulness, 5, 46-59. http://dx.doi.org/10.1007/s12671-012-0146-x

Braun, V., \& Clarke, V. (2006). Using Thematic Analysis in Psychology. Qualitative Research in Psychology, 3, 77-101. http://dx.doi.org/10.1191/1478088706qp063oa

Carlson, E. N. (2013). Overcoming the Barriers to Self-Knowledge: Mindfulness as a Path to Seeing Yourself as You Really Are. Perspectives on Psychological Science, 8, 173-186. http://dx.doi.org/10.1177/1745691612462584

Cebolla i Martí, A., \& Barrachina, M. T. M. (2009). The Effects of Mindfulness-Based Cognitive Therapy: A Qualitative Approach. Psychology in Spain, 13, 9-16.

Baer, R. A. (2003). Mindfulness Training as a Clinical Intervention: A Conceptual and Empirical Review. Clinical Psychology: Science and Practice, 10, 125-143. http://dx.doi.org/10.1093/clipsy.bpg015

Baer, R. A. (2009). Self-Focused Attention and Mechanisms of Change in Mindfulness-Based Treatment. Cognitive Behaviour Therapy, 38, 15-20. http://dx.doi.org/10.1080/16506070902980703

Brown, K. W., \& Ryan, R. M. (2003). The Benefits of Being Present: Mindfulness and Its Role in Psychological Well-Being. Journal of Personality and Social Psychology, 84, 822-848. http://dx.doi.org/10.1037/0022-3514.84.4.822

Finucane, A., \& Mercer, S. W. (2006). An Exploratory Mixed Methods Study of the Acceptability and Effectiveness of Mindfulness-Based Cognitive Therapy for Patients with Active Depression and Anxiety in Primary Care. BMC Psychiatry, 6, 1-14. http://dx.doi.org/10.1186/1471-244X-6-14

Grossman, P. (2008). On Measuring Mindfulness. Journal of Psychosomatic Research, 64, 405-408. http://dx.doi.org/10.1016/j.jpsychores.2008.02.001

Hayes, S. C. (2004). Acceptance and Commitment Therapy, Relational Frame Theory, and the Third Wave of Behavioural and Cognitive Therapies. Behaviour Therapy, 35, 639-665. http://dx.doi.org/10.1016/S0005-7894(04)80013-3

Ingvarsson, T., Nordén, T., \& Norlander, T. (2014). Mindfulness-Based Cognitive Therapy: A Case Study on Experiences of Healthy Behaviours by Clients in Psychiatric Care. Open Journal of Medical Psychology, 3, 390-402. http://dx.doi.org/10.4236/ojmp.2014.35041

Kabat-Zinn, J. (2003). Mindfulness-Based Interventions in Context: Past, Present and Future. Clinical Psychology: Science and Practice, 10, 144-156. http://dx.doi.org/10.1093/clipsy.bpg016

Kaplan, W., \& Laing, R. (2004). Priority Medicine for Europe and the World. Geneva: World Health Organization.

Kvale, S., \& Brinkmann, S. (2009). InterViews: Learning the Craft of Qualitative Interviewing (2nd ed.). Los Angeles, CA: Sage Publications.

Langdon, S., Jones, F. W., Hutton, J., \& Holttum, S. (2011). A Grounded-Theory Study of Mindfulness Practice Following Mindfulness-Based Cognitive Therapy. Mindfulness, 2, 270-281. http://dx.doi.org/10.1007/s12671-011-0070-5

Lau, M. A., \& Segal, Z. V. (2007). Mindfulness-Based Cognitive Therapy as a Relapse Prevention Approach to Depression. In K. A. Witkiewitz, \& G. A. Marlatt (Eds.), Therapist's Guide to Evidence-Based Relapse Prevention (pp. 73-90). London: Academic Press. http://dx.doi.org/10.1016/B978-012369429-4/50034-3

Lilja, J. L., Lundh, L-G., Josefsson, T., \& Falkenström, F. (2012). Observing as an Essential Facet of Mindfulness: A Comparison of FFMQ Patterns in Meditating and Non-Meditating Individuals. Mindfulness, 4, 203-212. http://dx.doi.org/10.1007/s12671-012-0111-8

Linehan, M. M. (1993). Cognitive-Behavioral Treatment of Borderline Personality Disorder. New York: Guilford Press.

Malpass, A., Carel, H., Ridd, M., Shaw, A., Kessler, D., Sharp, D., Bowden, M., \& Wallond, J. (2011). Transforming the Perceptual Situation: A Meta-Ethnography of Qualitative Work Reporting Patients' Experiences of Mindfulness-Based Approaches. Mindfulness, 3, 60-75. http://dx.doi.org/10.1007/s12671-011-0081-2

Malterud, K. (2001). Qualitative Research: Standards, Challenges and Guidelines. The Lancet, 358, 483-488. http://dx.doi.org/10.1016/S0140-6736(01)05627-6

Mason, O., \& Hargreaves, I. (2001). A Qualitative Study of Mindfulness-Based Cognitive Therapy for Depression. British Journal of Medical Psychology, 74, 197-212. http://dx.doi.org/10.1348/000711201160911

Michalak, J., Burg, J., \& Heidenreich, T. (2012). Don’t Forget Your Body: Mindfulness, Embodiment and the Treatment of Depression. Mindfulness, 3, 190-199. http://dx.doi.org/10.1007/s12671-012-0107-4

Munshi, K., Eisendrath, S., \& Delucchi, K. (2012). Preliminary Long-Term Follow-Up of Mindfulness-Based Cognitive 
Therapy-Induced Remission of Depression. Mindfulness, 4, 354-361. http://dx.doi.org/10.1007/s12671-012-0135-0

Nyklicek, I. (2010). Mindfulness, Emotion Regulation and Well-Being. In I. Nyclieck, A. Vingerhoets, \& M. Zeelenberg (Eds.), Emotion Regulation and Well-Being (pp. 101-118). New York: Springer Science and Business Media.

Segal, Z. V., Williams, J. M., Teasdale, J. D., \& Gemar, M. (1996). A Cognitive Science Perspective on Kindling and Episode Sensitization in Recurrent Affective Disorder. Psychological Medicine, 26, 371-380. http://dx.doi.org/10.1017/S0033291700034760

Segal, Z. V., Williams, J. M. G., \& Teasdale, J. D. (2013). Mindfulness-Based Cognitive Therapy for Depression (2nd ed.). New York: The Guilford Press.

Sobocki, P., Angst, J., Jönsson, B., \& Rehnberg, C. (2006). Cost of Depression in Europe. Journal of Mental Health Policy and Economics, 9, 87-98.

Spitzer, R. L., Williams, J. B., Kroenke, K., Linzer, M., deGruy III., F. V., Hahn, S. R., Brody, D., \& Johnson, J. G. (1994). Utility of a New Procedure for Diagnosing Mental Disorder in Primary Care. The PRIME-MD 1000 Study. Journal of the American Medical Association, 272, 1749-1756. http://dx.doi.org/10.1001/jama.1994.03520220043029

Teasdale, J. D., Segal, Z., \& Williams, J. M. G. (1995). How Does Cognitive Therapy Prevent Depressive Relapse and Why Should Attentional Control (Mindfulness) Training Help? Behaviour Research and Therapy, 33, 25-39.

http://dx.doi.org/10.1016/0005-7967(94)E0011-7 